\title{
Sliding Mode Control of the X-33 With an Engine Failure
}

\author{
Yuri B. Shtessel ${ }^{\dagger}$, Charles E. Hall ${ }^{\frac{1}{+}}$
}

\begin{abstract}
$\underline{\text { Abstract }}$
Ascent flight control of the $\mathrm{X}-33$ is performed using two XRS-2200 linear aerospike engines. in addition to atrusurtaces. The baseline control algorithms are PID with gain scheduling. Flight control using an innovative method. Sliding Mode Control. is presented for nominal and engine failed modes of flight. An easy to implement, robust controller. requiring no reconfiguration or gain scheduling is demonstrated through high fidelity flight simulations. The proposed sliding mode controller utilizes a two-loop structure and provides robust. de-coupled tracking of both orientation angle command profiles and angular rate command profiles in the presence of engine filure, bounded external disturbances (wind gusts) and uncertain matrix of inertia. Sliding mode control causes the angular rate and orientation angle tracking error dynamics to be constrained to linear, de-coupled, homogeneous, and vector valued difierential equations with desired eigenvalues. Conditions that restrict engine failures to robustness domain of the sliding mode controller are derived. Overall stability of a two-loop flight control system is assessed. Simulation results show that the designed controller provides robust, accurate, de-coupled tracking of the orientation angle command frofiles in the presence of external disturbances and vehicle inertia uncertainties. as well as the single engine failed case. The designed robust controller will significantly reduce the time and cost associated with nying new trajectory profiles or orbits, with new payloads, and with modified vehicles.
\end{abstract}

\section{Introduction}

Attitude control for the X-33 depends primarily on its two linear aerospike engines for ascent flight. Aerosurfaces are effective only during regions of flight with sufficient dynamic pressure. therefore the engines must proside all attitude control at liftoff. and the majority of the control at high altitudes. Ascent is quite demanding of the Ascent Flight Control System. or AFCS, due to the many trajectory requirements, operating constraints for the engines and areosurfaces, and the presence of high winds at the launch site. A thorough expose of the baseline X-33 attitude control system and description of the flight profile may be found in [13]. A description of the operation and control of the XRS-2200 linear aerospike engine may also be found in this reference.
The baseline AFCS. employs a variable structure PID control law [12] with gain scheduling. This requires four gains per channel that are looked up from a table as a function of relative velocity. Depending on the flight trajectory, each gain table can have as many as 25 values. so potentially 300 gain values must be stored in the on board computer for nominal flight. In case of an engine failure, or Power Pack Out (PPO) alternate sets of gain tables are used, depending on the flight time when the failure occurred. Provisions are made for 25 possible PPO times, or 25 sets of PID tables. This amounts to another 7500 values to be stored, or a total of 7800 values to provide gains for the nominal and engine failed cases.

\footnotetext{
${ }^{\dagger}$ Associate Professor, Electrical and Computer Engineering Dept., University of Alabama, Huntsville, Al.

‡ Aerospace Engineer, Control Systems Group, NASA, MSFC
}

Copyright (ㄷ) 2000 by the American Institute of Aeronautics and Astronautics, Inc. No copyright is asserted in the United States under Title 17, U.S. Code. The U.S. government has royalty-free license to exercise all rights under the copyright claimed herein for Governmental Purposes. All other rights are reserved under the copyright owner. 


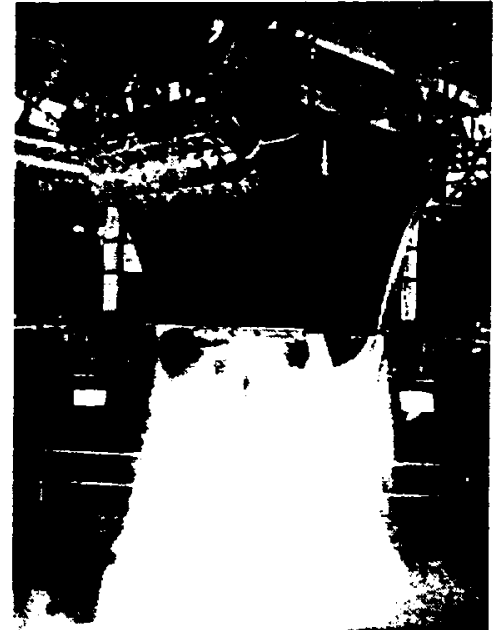

$\mathrm{X}-\mathbf{3 3}$ aerospike engine test firing at Stennis Space Center.

The reason for so many gain tables is because the control system design relies on linear analysis and perturbation theory at specific design points along the trajectory. This method is well established and has been used in many launch vehicle control system designs. therefore well understood and reliable tools and design methods may be used. Robust control is ensured as long as the vehicle performance and operating conditions are relatively close to the design points. For the $\mathrm{X}-33$, the vehicle performance and operating conditions as a function of relative velocity is very different between the first flight trajectory and the second. These design point parameters are drastically different with a failed engine, as can be seen in figures 1 and 2 . Therefore. alternate tables are needed for robust control to fly different trajectories. or to accommodate an engine failure at various times.

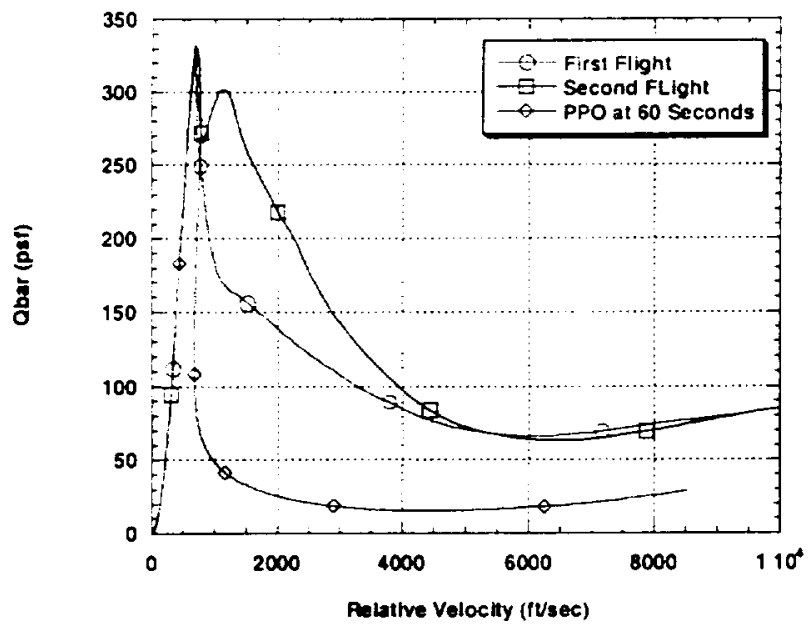

Figure 1: Dynamic pressure vs. relative velocity.

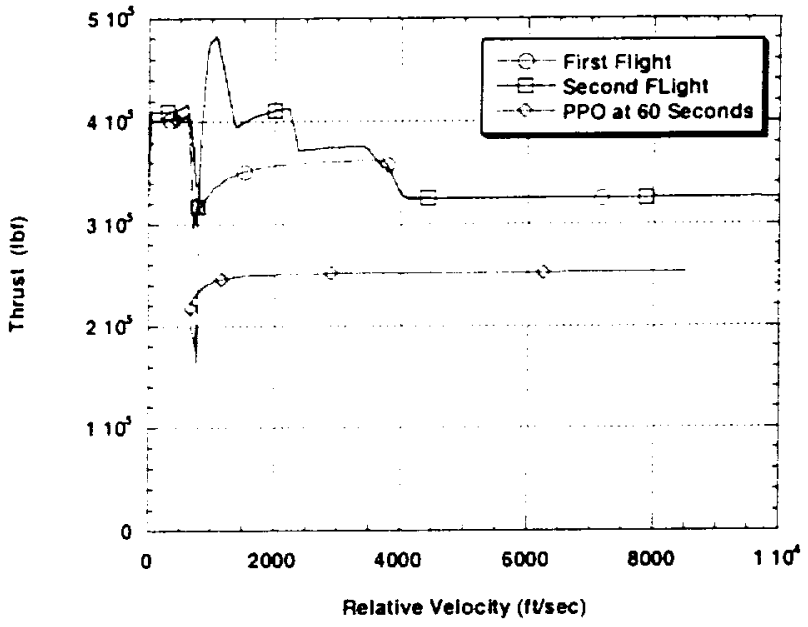

Figure 2: Thrust vs. relative velocity.

Flight control of the X-33 technology demonstration sub orbital launch vehicle in ascent mode involves attitude maneuvering through a wide range of flight conditions, wind disturbances and uncertainties of the X-33 mathematical model, including uncertain matrix of inertia and an engine failure. A robust flight control algorithm that would accommodate different trajectories and an engine failure without gain scheduling would be an improvement over the X-33 current flight control technology. Sliding Mode Controller (SMC) is an attractive robust control algorithm for the $\mathrm{X}-33$ ascent designs because of its inherent insensitivity and robustness to plant uncertainties and external disturbances ${ }^{1-3}$. Such a controller would drastically decrease the amount of time spent in pre-flight analysis, thus reducing cost.

A SMC design consists of two major steps: (1) a sliding surface is designed such that the system motion on this surface exhibits desired behavior in the presence of plant uncertainties and disturbances; and (2) a control function is designed that causes the system state to reach the sliding surface in finite time and guarantees system motion in this surface thereafter. The system's motion on the sliding surface is called sliding mode. Strict enforcement of the sliding mode typically leads to discontinuous control functions and possible control chattering effects ${ }^{4}$. Control chattering is typically an unwanted effect that is easily eliminated by continuous approximations of discontinuous control functions $s^{4,5}$, or by continuous SMC designs ${ }^{6}$. 
An example of the application of SMC is shown in the spacecraft attitude control work performed by Dwyer et al'. This work utilizes the natural time-siale separation. which exists in the system dynamics of many aerospace control problems".

The X-33 SMC architecture. which is developed in the paper'. is a two-loop structure that incorporates backstepping techniques ${ }^{10}$. In the outer loop, the kinematics equation of angular motion is used with the outer loop SMC to generate the angular rate protiles as virtual control inputs to the inner loop. In the inner loop, a suitable inner loop S.MC is designed so that the commanded angular rate profiles are tracked. The inner loop S.IC produces roll, pitch and yaw torque commands, which are optimally allocated into end-effector deflection commands. Multiple time scaling (multiple-scale) is defined as the timeconstant separation between the two loops. That is. the inner loop compensated dynamics is designed to be faster than the outer loop dynamics. The resulting multiple-scale two-loop SMC, with optimal torque allocation. causes the angular rate and the Euler angle tracking errors to be constrained to linear de-coupled homogeneous vector valued differential equations with desired eigenvalues placement.

Simulation results are presented that demonstrate the SMC's effectiveness in causing the $X-33$ technology demonstration vehicle. operating in ascent mode, to robustly follow the desired profiles. The resulting design controls large attitude maneuvers through a wide range of flight conditions, provides highly accurate tracking of guidance trajectories, and exhibits robustness to external disturbances. parametric uncertainties, and an engine failure.

\section{Equations of the $\mathrm{X}-33$ dynamics}

The dynamic equations of rotational motion of the $\mathrm{X}-33$ rigid-body are given by the Euler equation in the body frame

$$
\left(\mathbf{J}_{0}+\Delta \mathbf{J}\right) \dot{\omega}=-\Omega\left(\mathbf{J}_{0}+\Delta \mathbf{J}\right) \omega+\mathbf{T}+\mathbf{d}
$$

where $\mathbf{J}_{0} \in \mathbf{R}^{3 \times 3}$ is the nominal inertia matrix, $\Delta \mathbf{J} \in \mathbf{R}^{3 \times 3}$ is an uncertain part of the inertia matrix, caused by fuel consumption and variations of particular payloads from a nominal one. $\omega=\left[\begin{array}{lll}p & q & r\end{array}\right]^{T}$ is the angular rate vector, $\mathbf{T}=\{L, M, N\}^{r}$ is the control torque vector, $\mathbf{d}=\left\{L_{d}, M_{d}, N_{d}\right\}^{T}$ is the external disturbance torque vector. The matrix $\Omega$ is given by:

$$
\Omega=\left[\begin{array}{ccc}
0 & -\omega_{3} & \omega_{2} \\
\omega_{3} & 0 & -\omega_{1} \\
-\omega_{2} & \omega_{1} & 0
\end{array}\right] .
$$

The X-33 orientation dynamics are described by the kinematics equation on the body axes

$\dot{\gamma}=\mathbf{R}(\gamma) \omega$,

where

$$
\left\{\begin{array}{l}
\mathbf{R}(\gamma)=\left[\begin{array}{lll}
1 & \tan \theta \sin \varphi & \tan \theta \cos \varphi \\
0 & \cos \varphi & -\sin \varphi \\
0 & \frac{\sin \varphi}{\cos \theta} & \frac{\cos \varphi}{\cos \theta}
\end{array}\right] \\
\gamma=\left[\begin{array}{lll}
\varphi & \theta & \psi
\end{array}\right]^{T}
\end{array}\right.
$$

The equations of the X-33 translational motion are available as well ${ }^{9}$ but not presented in the paper, because they do not effect the orientation angle controller design. However, these equations are used during 6DOF high fidelity simulations to assess performance of the designed sliding mode controller.

The control torque $\mathbf{T}$ is generated by the aerodynamic surfaces and rocket engines. This is described by the equation

$$
\mathbf{T}=\mathbf{D}(.) \delta
$$

where $\mathbf{D}(.) \in \mathbf{R}^{3 x n}$ is a sensitivity matrix calculated on the basis of table lookup data, $\delta \in \mathbf{R}^{n}$ is the vector of aerodynamic surface deflections and differential throttles of the rocket engines.

Electromechanical actuators deflect the aerodynamic surfaces (aerosurfaces), and thrust- 
vector-control valves that throttle the rocket engines. executing the following commands

$\delta_{i}=\mathbf{D}^{*}(.) \mathbf{T}$.

where a control allocation matrix $\mathbf{D}^{\sharp}($.$) that meets a$ pseudo-inverse condition $\mathbf{D}(.) \mathbf{D}(.)^{\#}=\mathbf{I}$ can be calculated as follows:

$\mathbf{D}^{*}()=.\mathbf{D}(.)^{\mathrm{T}}\left[\mathbf{D}(.) \mathbf{D}(.)^{\mathrm{T}}\right]^{-1}$

The actuator dynamics are assumed to be much faster than the dynamics of the compensated $X-33$ flight control system that permits to assume $\delta \approx \delta_{c}$. The "fast" actuator dynamics are neglected at the stage of the controller design. They will be used during simulations to validate the designed controller. Taking into account possible engine failures, the actual sensitivity matrix must be rewritten. This is

$\mathbf{D}_{a c t}()=.\mathbf{D}()+.\Delta \mathbf{D}($.$) .$

where $\Delta \mathbf{D}($.$) is an uncertain component of the$ sensitivity matrix due to possible engine failures, while the allocation matrix $D^{*}($.$) is still calculated$ on the basis of the nominal sensitivity matrix in accordance with eq. (5b). Taking into account eqs. (5a) and $(5 c)$ the control torque in eq. (5) must be recalculated as

$\mathbf{T}_{c}=(\mathbf{D}()+.\Delta \mathbf{D}().) \delta_{c}$.

where $\mathbf{T}_{c}$ stands for the actual control torque generated by the deflections $\delta_{c}$.

Substituting eq. (5a) into the eq. (5d) we obtained a final expression for the actual control torque

$\mathbf{T}_{\boldsymbol{c}}=(\mathbf{I}+\mathbf{E}) \mathbf{T}$,

where $\mathbf{E}=\ddot{\mathbf{A}} \mathbf{D}(.) \mathbf{D}^{*}$.

\section{Problem Formulation}

The problem formulation is to determine the actuator deflection commands $\delta$, such that the commanded Euler angle profiles $\gamma_{i}=\left[\begin{array}{lll}\varphi_{c} & \theta_{c} & \psi_{i}\end{array}\right]^{T}$ are robustly followed in the presence of bounded disturbance torque $\mathbf{d}$, the $\mathrm{X}-33$ inertia variations $\Delta \mathbf{J}$ and engine failures that are described by the uncertain matrix $\Delta D($.$) .$

The control problem for the $\mathrm{X}-33$ in ascent or launch mode is to determine the control torque input command vector $\mathbf{T}$ in the given state variable equations

$\left(\mathbf{J}_{0}+\Delta \mathbf{J}\right) \dot{\omega}=-\Omega\left(\mathbf{J}_{0}+\Delta \mathbf{J}\right) \boldsymbol{\omega}+(\mathbf{I}+\mathbf{E}) \mathbf{T}+\mathbf{d}$ (7a)

$\dot{\gamma}=\mathbf{R}(\gamma) \omega$

$\mathbf{y}=\gamma$

where $\mathbf{E}=\ddot{\mathbf{A}} \mathbf{D}(.) \mathbf{D}^{*}$, such that the output vector $\mathbf{y}$ asymptotically tracks a command orientation angle profile $\mathbf{y}_{r}=\gamma_{c}$. That is,

$\lim _{i \rightarrow \infty}\left\|y_{i r}(t)-y_{i}(t)\right\|=0 \quad \forall i=\overline{1,3}$

The desired $\mathrm{X}-33$ performance criteria is to robustly track both the commanded Euler angle guidance profiles $\gamma_{c}$ and the real-time generated angular rate profiles $\omega_{c}$, such that the motion for each quantity is described by a linear, de-coupled, and homogeneous vector valued differential equations with given eigenvalues.

\section{Sliding Mode Controller Design}

Tracking of the commanded Euler angles guidance profile $\gamma_{c}$ (the primary objective) is achieved through a two loop SMC structure?. The cascade structure of the system in eq. (7) and the inherent two time scale nature of the $X-33$ flight control problem are exploited for design of a two loop flight control system using continuous SMCs in the inner and outer loops. The outer loop SMC 
provides angular rate commands $\omega_{i}$, to the inner

loop. The inner loop SMC provides robust decoupled tracking of angular rates $\omega$. Together, the inner and outer loop SMCs form a wo loop tlight control system that achieves de-coupled asymptotic tracking of the command angle guidance protile $\gamma_{\text {: }}$

Similar structure was developed for an aircraft control system using a dynamic inversion algorithm. which, however, does not exhibit very robust behavior.

\section{Outer Loop Sliding Mode Controller Design}

The outer loop SMC takes the $\mathrm{X}-33$ angular rate vector $\omega$ as a virtual control $\omega_{c}$. and uses the kinematics eq. (7b) to compensate the Euler angle tracking dynamics. The motion of the outer loop compensated tracking error dynamics, with desired bandwidth, is constrained (by proper control action) to a sliding surface of the form

$\sigma=\gamma_{e}+\mathbf{K}_{1} \int_{0}^{1} \gamma_{e} d \tau, \quad \sigma \in \mathbf{R}^{3}$.

where

$$
\gamma_{e}=\gamma_{c}-\gamma
$$

$\mathbf{K}_{1}=\operatorname{diag}\left\{k_{\mathrm{I}_{1}}\right\}, \mathbf{K}_{1} \in \mathbf{R}^{3 \times 3}$. The gain matrix

$\mathbf{K}_{\text {I }}$ is chosen so that the output tracking error $\gamma_{e}$ exhibits a desired linear asymptotic behavior on the sliding surface ( $\sigma=0$ ).

The objective of the outer loop SMC is to generate the commanded angular rate vector $\omega_{c}$ (which is passed to the inner loop SMC) necessary to cause the vehicle's trajectory to track the commanded Euler angles guidance profile $\gamma_{c}$. In other words, the virtual control law $\omega_{c}$ is designed to provide asymptotic convergence (with finite reaching time) of the system's eq. (7b) trajectory to the sliding surface $\sigma=0$. Dynamics of the sliding surface in eq. (9) are described as

$\dot{\sigma}=\dot{\gamma}_{c}-\mathbf{R}(\gamma) \omega_{c}+\mathbf{K}_{1} \gamma_{e}$

The outer loop SMC design is initiated by choosing a candidate Lyapunov function of the form

$V=\frac{1}{2} \sigma^{r} \sigma>0$ whose derivative is shown as

$$
\dot{V}=\sigma^{T} \dot{\sigma}=\sigma^{T}\left[\dot{\gamma}_{c}-\mathbf{R}(\gamma) \omega_{c}+\mathbf{K}_{1} \gamma_{e}\right]
$$

To ensure asymptotic stability of the origin of the system in eq. (10), the following derivative inequality of the candidate Lyapunov function is enforced ${ }^{1-4}$

$$
\dot{V}=-\rho \sigma^{T} \operatorname{SIGN}(\sigma)=-\rho \sum_{i=1}^{3}\left|\sigma_{i}\right|, \quad \rho>0 \text {. }
$$

Considering eq. (13), the required angular rate command $\omega_{c}$ to ensure asymptotic stability is defined as

$$
\omega_{c}=\mathbf{R}^{-1}(\gamma)\left[\dot{\gamma}_{c}+\mathbf{K}_{1} \gamma_{e}\right]+\mathbf{R}^{-1}(\gamma) \rho \operatorname{SIGN}(\sigma)
$$

where

$\operatorname{SIGN}(\sigma)=\left[\operatorname{sign}\left(\sigma_{1}\right), \operatorname{sign}\left(\sigma_{2}\right), \operatorname{sign}\left(\sigma_{3}\right)\right]^{T}$, and $\rho>0$.

The sliding surface shown in eq. (9) will reach zero in a finite time $\mathrm{l}^{1-4}$ defined as

$t_{r}=\max _{i \in[1.3]} \frac{\left|\sigma_{i}(0)\right|}{\rho}$,

where $\rho>0$, and $t_{r}$ is the design parameter describing the sliding surface reaching time. The value of $\rho$ is calculated using eq. (15).

The angular rate command profile in eq. (14) is discontinuous; as a result, the control actuation will chatter during system operation on the sliding surface eq. (9). Mechanically and electrically, this chattering is an unwanted effect. Moreover, a discontinuous profile cannot be accurately tracked in the inner loop. To solve this problem, the discontinuous term ( $\operatorname{SIGN}(\sigma)$ ) in eq. (14) is replaced by the high-gain linear term 
$\mathbf{K}_{0} \sigma=\left\{\frac{\sigma_{1}}{\varepsilon_{1}}, \frac{\sigma_{2}}{\varepsilon_{2}}, \frac{\sigma_{3}}{\varepsilon_{3}}\right\}^{T}$,

$\mathbf{K}_{\nu}=\operatorname{diag}\left\{\frac{1}{\varepsilon_{i}}\right\} \quad \forall \varepsilon_{1}>0 \div \overline{1.3}$

where $\varepsilon_{1}>0 \forall \overline{1.3}$ define the slopes of the linearized function ${ }^{45}$.

After substitution of eqs. $(161$ and $(1+)$ into eq. (10). the sliding surtace dynamics are of the form

$\dot{\sigma}=-\rho \mathbf{K}_{0} \sigma$

Eq. (17) is globally asymptotically stable, since $\rho>0$ and $K_{0}$ is positive definite, and the equilibrium $\sigma=0$ will be reached asymptotically. Moreover, in a close vicinity of $\sigma_{i}=0, \forall i=\overline{1,3}$, the tracking error $\gamma_{e}$ will exhibit de-coupled motion in accordance with eq. (9).

\section{Inner Loop Sliding Mode Controller Design}

The purpose of the inner loop SMC is to generate the vehicle torque command vector $\mathbf{T}$ necessary to track the given commanded angular rate profile $\omega_{\text {. }}$. In addition to solving the inner loop tracking problem defined as

$\lim _{t \rightarrow \infty}\left\|\omega_{n}(t)-\omega_{1}(t)\right\|=0, \forall i=1.3$

SMC causes the system to exhibit linear decoupled motion in sliding mode.

The motion of the inner loop compensated dynamics, with desired bandwidth. is constrained (by proper control action) to a sliding surface of the form

$$
s=\omega_{e}+\mathbf{K}_{2} \int_{0}^{t} \omega_{e} d \tau, \quad s \in \mathbf{R}^{\vdots}
$$

where

$$
\omega_{e}=\omega_{c}-\omega
$$

$\mathbf{K}_{2}=\operatorname{diag}\left\{k_{2}\right\}, \mathbf{K}_{2} \in \mathbf{R}^{3 \times 3}$. The angular rate tracking error $\omega_{e}$ exhibits a desired linear asymptotic behavior while operating on the sliding surface ( $s=0$ ) and with proper choice of $\mathbf{K}_{2}$. The inner loop sliding mode dynamics in eq. (19) are designed faster than the outer-loop sliding mode dynamics in eq. (9) in order to preserve sufficient time scale separation between the loops.

The command torque $\mathbf{T}$ is designed to provide asymptotic convergence (with finite reaching time) of the system's eq. (7a) trajectory to the sliding surface $s=0$. Dynamics of the sliding surface eq. (19) are described as follows:

$$
\begin{aligned}
& \dot{s}=\dot{\omega}_{c}+\left(\mathbf{J}_{0}+\Delta \mathbf{J}\right)^{-1} \Omega\left(\mathbf{J}_{0}+\Delta \mathbf{J}\right) \omega- \\
& \left(\mathbf{J}_{0}+\Delta \mathbf{J}\right)^{-1}(\mathbf{I}+\mathbf{E}) \mathbf{T}_{c}-\left(\mathbf{J}_{0}+\Delta \mathbf{J}\right)^{-1} \mathbf{d}+\mathbf{K}_{2} \omega_{e}
\end{aligned}
$$

Assuming the matrix $\mathbf{I}+\mathbf{E}$ is positive definite, that can be achieved if $\sum_{j=1}^{n}|E|_{i j}<1 \quad \forall i=\overline{1, n}$, and considering a fact that the inertia matrix $\mathbf{J}_{0}+\Delta \mathbf{J}$ is positive definite. the inner loop SMC design is initiated by choosing the candidate Lyapunov function of the form

$$
V=\frac{1}{2} s^{T}(\mathbf{I}+\mathbf{E})^{-1}\left(\mathbf{J}_{0}+\Delta \mathbf{J}\right) s>0
$$

whose derivative is shown as

$$
\begin{aligned}
& \dot{V}=\left\{s^{\top} \frac{1}{2}(\mathbf{I}+\mathbf{E})^{-1}\left[\Delta \mathbf{J}-\dot{\mathbf{E}}(\mathbf{I}+\mathbf{E})^{-1}\right] s+\right. \\
& (\mathbf{I}+\mathbf{E})^{-1}\left(\mathbf{J}_{0}+\Delta \mathbf{J}\right) \dot{\omega}_{c}+(\mathbf{I}+\mathbf{E})^{-1}\left(\mathbf{J}_{0}+\Delta \mathbf{J}\right) \mathbf{K}_{2} \omega_{c}+ \\
& \left.(\mathbf{I}+\mathbf{E})^{-1} \Omega\left(\mathbf{J}_{0}+\Delta \mathbf{J}\right) \omega-\mathbf{T}_{c}-(\mathbf{I}+\mathbf{E})^{-1} \mathbf{d}\right\}
\end{aligned}
$$

To ensure asymptotic stability of the origin of the system eq. (20), the following derivative of the Lyapunov function candidate is enforced ${ }^{1-4}$

$$
\dot{V} \leq-\eta s^{T} \operatorname{SIGN}(s)=-\eta \sum_{i=1}^{3}\left|\sigma_{i}\right|, \quad \eta>0 .
$$

Further, the sliding surface eq. (19) will reach zero in a finite time ${ }^{1-4}$ defined by

$$
\tau_{r} \leq \max _{i \in[1,3]} \frac{\left|s_{i}(0)\right|}{\eta}
$$


where $\eta>0$, and $\tau_{\text {r }}$ is the design parameter describing the sliding surface reaching time. The value of $\eta$ is calculated using inequality (24).

Considering inequality $(23)$, the required torque command $\mathbf{T}$ to ensure asymptotic stability is defined as

$\mathbf{T}=\mathbf{J}_{0} \dot{\omega}_{r}+\mathbf{J}_{0} \mathbf{K}_{2} \omega_{e}+\Omega \mathbf{J}_{0} \omega+\frac{1}{2} \mathrm{M} s+$ $\tilde{\rho} \operatorname{SIGN}(s)$

where $\mathrm{M}=\operatorname{diag}\left\{\mu_{1}\right\}, \mu_{1}>0 \quad \forall i=\overline{1,3}$. One should note that the SMC eq. (25) is independent of uncertainties $\Delta \mathbf{J} . \Delta \dot{\mathbf{J}}, \mathbf{E}, \dot{\mathbf{E}}$ and disturbances $\mathbf{d}$. Substituting eq. (25) into eq. (22) yields

$$
\begin{aligned}
& \dot{V}= \\
& -\tilde{\rho} \sum_{i=1}^{3}\left|s_{i}\right|-\frac{1}{2} s^{T}\left\{\mathbf{M}-(\mathbf{I}+\mathbf{E})^{-1}\left[\begin{array}{l}
\Delta \mathbf{J}- \\
\dot{\mathbf{E}}(\mathbf{I}+\mathbf{E})^{-1}
\end{array}\right]\right\} s+ \\
& s^{T}\left[\left((\mathbf{I}+\mathbf{E})^{-1}\left(\mathbf{J}_{0}+\Delta \mathbf{J}\right)-\mathbf{J}_{0}\right) \dot{\omega}_{i}+\right. \\
& \left((\mathbf{I}+\mathbf{E})^{-1}\left(\mathbf{J}_{0}+\Delta \mathbf{J}\right)-\mathbf{J}_{0}\right) \mathbf{K}_{2} \omega_{e}+ \\
& \left.\left((\mathbf{I}+\mathbf{E})^{-1} \Omega\left(\mathbf{J}_{0}+\Delta \mathbf{J}\right)-\Omega \mathbf{J}_{0}\right) \omega-(\mathbf{I}+\mathbf{E})^{-1} \mathbf{d}\right]
\end{aligned}
$$$$
\text { (26) }
$$

Selecting the matrix $\mathrm{M}=\operatorname{diag}\left\{\mu_{i}\right\}, \mu_{i}>0 \forall i=\overline{1.3}$ to diagonally dominate the matrix $(\mathbf{I}+\mathbf{E})^{-1}\left(\Delta \mathbf{J}-\dot{\mathbf{E}}(\mathbf{I}+\mathbf{E})^{-1}\right)$ we ca find a positive definite matrix $\mathbf{Q}$ such that $s^{T} \mathbf{Q} s \geq s^{T}\left\{\mathbf{M}-(\mathbf{I}+\mathbf{E})^{-1}\left\lfloor\Delta \dot{\mathbf{J}}-\dot{\mathbf{E}}(\mathbf{I}+\mathbf{E})^{-1}\right\} \hat{\beta}\right.$.

Assuming

$$
\begin{aligned}
& \left|\left[\left((\mathbf{I}+\mathbf{E})^{-1}\left(\mathbf{J}_{0}+\Delta \mathbf{J}\right)-\mathbf{J}_{0}\right) \dot{\omega}_{i}\right]_{i}\right|<a_{i}, \\
& \left|\left[\left((\mathbf{I}+\mathbf{E})^{-1}\left(\mathbf{J}_{0}+\Delta \mathbf{J}\right)-\mathbf{J}_{0}\right) \mathbf{K}_{2} \omega_{e}\right]_{i}\right|<b_{i}, \\
& \left|\left[\left((\mathbf{I}+\mathbf{E})^{-1} \Omega\left(\mathbf{J}_{0}+\Delta \mathbf{J}\right)-\Omega \mathbf{J}_{0}\right) \omega\right]_{i}\right|<c_{i}, \\
& \left|\left[(\mathbf{I}+\mathbf{E})^{-1} \mathbf{d}\right]_{i}\right|<L_{i}
\end{aligned}
$$

we can rewrite eq. (26) as follows:

$$
\begin{aligned}
& \dot{V} \leq-\sum_{i=1}^{3}\left(\tilde{\rho}-a_{i}-b_{i}-c_{i}-L_{i}\right)\left|s_{i}\right|-\frac{1}{2} s^{T} \mathbf{Q} s \leq \\
& -\sum_{i=1}^{3}\left(\tilde{\rho}-a_{i}-b_{i}-c_{i}-L_{i}\right)\left|s_{i}\right|
\end{aligned}
$$

Inequality (28) is rewritten to enforce inequality (23) after selecting $\mu>\lambda$. This is.

$\dot{V} \leq-\sum_{i=1}^{3}\left(\widetilde{\rho}-a_{i}-b_{i}-c_{i}-L_{i}\right)\left|s_{i}\right| \leq-\eta \sum_{i=1}^{3}\left|s_{i}\right|$

The value of parameter $\widetilde{\rho}$ is identified in accordance with inequality (29) as follows:

$\tilde{\rho} \geq a_{i}+b_{i}+c_{i}+L_{i}+\eta$

Remark. The constants $a_{i}, b_{i}, c_{i}, L_{i} \forall i=\overline{1,3}$, are derived by estimating the upper bounds of inequalities (27) within a reasonable flight domain.

To avoid chattering, the SMC eq. (25) is implemented in a continuous form

$$
\mathbf{T}_{c}=\mathbf{J}_{0} \dot{\omega}_{c}+\mathbf{J}_{0} \mathbf{K}_{2} \omega_{\epsilon}+\Omega \mathbf{J}_{0} \omega+\frac{1}{2} \mathbf{M} s+\tilde{\rho} S A T(\Gamma s)
$$

where

$$
\Gamma=\operatorname{diag}\left\{\frac{1}{\zeta_{i}}\right\} \forall i=\overline{1,3},
$$$$
\operatorname{SAT}(\Gamma s)=\left\{s a t \frac{s_{1}}{\zeta_{1}}, \text { sat } \frac{s_{2}}{\zeta_{2}}, \text { sat } \frac{s_{3}}{\zeta_{3}}\right\}^{T},
$$$$
\text { sat } \frac{s_{i}}{\zeta_{i}}= \begin{cases}1, & \text { if } s_{i}>\zeta_{i} \\ \frac{s_{i}}{\zeta_{i},} & \text { if }\left|s_{i}\right| \leq \zeta_{i} \\ -1, & \text { if } s_{i}<-\zeta_{i}\end{cases}
$$ 
by the first three equations in eqs. (36) are bounded with boundary widths proportional to $\zeta, \forall i=\overline{1.3}$.

\section{Design and Simulation of the $\mathrm{X}-33$ Control System in Ascent Mode}

The X-33 technology demonstration vehicle controller design is considered in ascent mode. The 6DOF high fidelity mathematical model includes the control deflection vector $\delta \in \mathbf{R}^{11}$ that consists of the following components: $\delta_{1}$ and $\delta_{2}$ are deflections of the right and left tlaps. $\delta_{3}$ and $\delta_{4}$ are deflections of the right and left inboard elevons, $\delta_{5}$ and $\delta_{6}$ are deflections of the right and left outboard elevons; $\delta_{7}$ and $\delta_{8}$ are deflections of the right and left rudders; $\delta_{9}, \delta_{10}$, and $\delta_{11}$ are pitch, roll and yaw differential throttles (in $\%$ of power level) of the aerospike rocket engine. Flight guidance tables for the Euler angle command profiles $\gamma_{c}$ are used. High fidelity quantized sensor mathematical models with $0.02 \mathrm{~s}$ time delay as well as realistic table look up wind gust profiles that create a disturbance torque are also used.

The command profiles $\omega_{c}$ and $T$ are generated by the outer and inner loop SMCs in eqs. (14), (16) and (31). The deflection command vector $\delta_{r}$ is calculated in eq. (6) and executed by the actuators, whose dynamics are contained in high fidelity models used in the simulations.

The outer loop continuous SMC is designed in accordance with eqs. (9), (14) and (16). The following outer loop SMC parameters are chosen:

$$
\rho=1
$$$$
\mathbf{K}_{0}=\left[\begin{array}{ccc}
0.8 & 0 & 0 \\
0 & 0.9 & 0 \\
0 & 0 & 0.9
\end{array}\right]
$$

$$
\mathbf{K}_{1}=\left[\begin{array}{ccc}
0.3 & 0 & 0 \\
0 & 0.3 & 0 \\
0 & 0 & 0.3
\end{array}\right]
$$

where the matrix $\mathbf{K}_{1}$ provides a $0.3 \mathrm{rad} / \mathrm{s}$ given bandwidth for the compensated outer loop. The inner loop continuous SMC is designed in accordance with eqs. (19). (30) and (31). The following inner loop SIIC parameters are chosen:

$$
\hat{\rho}=\left[\begin{array}{lll}
1.5 \cdot 10^{6} & 0 & 0 \\
0 & 1.8 \cdot 10^{6} & 0 \\
0 & 0 & 2.0 \cdot 10^{6}
\end{array}\right] \quad N \cdot \mathrm{m} .
$$$$
\mathbf{K}_{:}=\left[\begin{array}{ccc}
1.0 & 0 & 0 \\
0 & 1.0 & 0 \\
0 & 0 & 1.0
\end{array}\right], \xi_{1}=1.0 \forall i=\overline{1.3} \text {. }
$$

where the matrix $\mathbf{K}_{2}$ provides a $1.0 \mathrm{rad} / \mathrm{sec}$ given bandwidth for the compensated inner loop. and $M=0$.

A high fidelity six degree-of-freedom flight simulation was used to test the performance of the SMC for nominal flight and with an engine failure, PPO at seconds. The simulation, called Maveric (Marshall Aerospace Vehicle Representation In C), is the same one used for $\mathrm{X}-33$ flight analyses. modified to accept the SMC. The same flex filters used in the baseline controller were used in the SMC. Maveric employs detailed, non-linear models for the two aerospike engines, electro-mechanical actuators for the aerosurfaces with associated linkages, and Inertial Navigation Unit (INU). All flight software is emulated, and data latency from unmodeled computations in the INU and in the data bus are simulated. Forces and torques from propellant slosh is included, as well as a mean annual wind profile at Edwards Air Force Base, where the X-33 will be launched.

Should one of the X-33's two engines fail, they are capable of re-configuring such that propellant flow can be diverted from the remaining functional engine, to the thrust cells of the failed engine. This is called Power Pack Out (PPO) mode. When one engine fails, the Gas Generator and turbo pumps in the failed engine are shut down. Then, two interengine isolation valves, one for fuel and the other for oxidizer, are opened allowing propellant flow from the functional engine to the thrust cells in the failed engine. In this way, both engines can provide thrust and thrust vector control as before, only at half the 
nominal level. The PPO mode was simulated at sec in flight. The sensed orientation angle tracking errors are shown in fig. 15 .

The 6DOF time simulation results for the first tlight guidance trajectory are presented in figures 3 - 17. Figures 1 - 3 show overall tracking pertormance of the Euler angle guidance profiles in the outer loop. which is obviously decoupled and very accurate. Figure 4 confirms existence of a sliding mode in the outer loop, since the Euler angle tracking happens in a close-to-zero vicinity of the outer loop sliding surfaces. Figures 5-7 show reasonably accurate tracking the angular rates command profiles in the inner loop. A piece-wise constant shape of the sensed rate profiles that are displayed in figures 6 and 7 is caused by quantization of sensored data from gyros. It's also worth noticing that in figure 8 the sliding surface $2\left(s_{2}\right)$ deviates significantly from zero still being limited by the boundary layer: $\left|s_{2}\right| \leq \zeta_{2}=1.0$. This deviation is due to a large disturbance in the pitch channel and can be estimated by eq. (36). Figure 9 demonstrates torque command profiles $\mathbf{T}$. These commands are allocated into actuator deflection $s \delta_{c}$, and rocket engine throttle commands executed by the actuators. Figures 10-14 demonstrate corresponding engine differential throttles and sensed deflections of the aerodynamic surfaces that are far from saturation.

\section{Conclusions}

Employing a time scaling concept in the inner (angular rates) and outer (orientation angles) loops, a new two-loop continuous sliding mode controller was designed for the $\mathrm{X}-33$. This sliding mode controller provides de-coupled performance in the inner and outer loops in presence of bounded external disturbances and plant uncertainties. Stability of the two-loop control system is analyzed. A control allocation matrix is designed that optimally distributes the roll, pitch, and yaw torque commands into end-effector deflection commands. A six degree of freedom high fidelity simulation shows that the multiple scale sliding mode controller provides robust, accurate, de-coupled tracking of the commanded Euler angle guidance profiles for the $\mathrm{X} 33$ in ascent mode in the presence of external disturbances (wind gusts) and plant uncertainties (changing matrix of inertia) and for the engine tailure. The designed robust controller is expected to significantly reduce the time and cost associated with tlying to new orbits, with new payloads, and with moditied vehicles. and to increase their safety and reliability. This is a significant advancement in performance over that typically achieved with gain scheduled control systems currently being used for launch vehicles.

\section{References}

1) DeCarlo, R. A., Zak, S. H.. and Matthews. G. P. "Variable structure control of nonlinear multivariable systems: a tutorial," IEEE Proceedings, Vol. 76, 1988, pp. 212-232.

2) Utkin, V. I., Sliding Modes in Control and Optimization, Berlin, Springer - Verlag, 1992, pp. 1582.

3) Hung, J. Y., Gao, W., and Hung, J. C., "Variable Structure Control: A Survey," IEEE Transactions on Industrial Electronics, Vol. 40 , No.1, 1993, pp. 2-21.

4) Slotine, J-J., and Li, W., Applied Nonlinear Control, Prentice Hall, New Jersey, 1991, pp. 211250.

5) Esfandiari, F., and Khalil, H.K., "Stability Analysis of a Continuous Implementation of Variable Structure Control," IEEE Transactions on Automatic Control, Vol. 36, No. 5, 1991, pp. 616-619.

6) Shtessel, Y., and Buffington, J., "Finitereaching-time continuous sliding mode controller for MIMO nonlinear systems," Proceedings of the Conference on Decision and Control, Vol.3, IEEE. 1998, pp. 1934-1935.

7) Dwyer, T. A. W., III, and Sira-Ramirez, H., "Variable Structure Control of Spacecraft Attitude Maneuvers," Journal of Guidance, Control and Dynamics, Vol. 11, No. 3, 1988, pp. 262-270.

8) Naidu, D. S., and Calise A. J., "Singular Perturbations and Time Scales in Guidance, Navigation, and Control of Aerospace Systems: Survey," Proceedings of the AlAA Guidance, Navigation, and Control Conference, Vol. 3, AIAA, 1995. pp. 1338-1362.

9) Shtessel, Y.. McDuffie, J., Jackson, M., Hall, C., Krupp, D., Gallaher, M., and Hendrix, N., "Sliding Mode Control of the X-33 Vehicle in Launch and Re-entry Modes," Proceedings of the AlAA Guidance, Navigation, and Control Conference, Vol. 3, AIAA, 1998, pp.1352-1362. 
10) Krstic, M., Kanellakopoulus, I., and Kokotovic. P. Nonlinear and Adaptive Control Design. John Wiley and Sons. NY. 1995. pp. 21-54.

11) Azam. M.. and Singh. S.. "Invertibility and Trajectory Control for Nonlinear Maneuvers of Aircraft." Journal of Guidance. Control, and Dynamic. Vol. 17. No. 1, 1994. pp. $192-$ 200.

12) Hall. C. E.. Hodel, A. S., and Hung. J. Y.."Variable Structure PID Control to Prevent Integral Windup", Proceedings of the $31^{\text {st }}$ Southeastern Symposium on System Theory. IEEE. 1999. pp. 169-173.

13) Hall, C. and Panossian. H., "X-33 Altitude Control Using the XRS-2200 Linear Aerospike Engine". Proceedings of the $35^{\text {th }}$ Annual AIAA/ASME/SAE/ASEE Joint Propulsion Conference and Exhibit, 20-23 June, 1999, Los Angeles. Ca. 


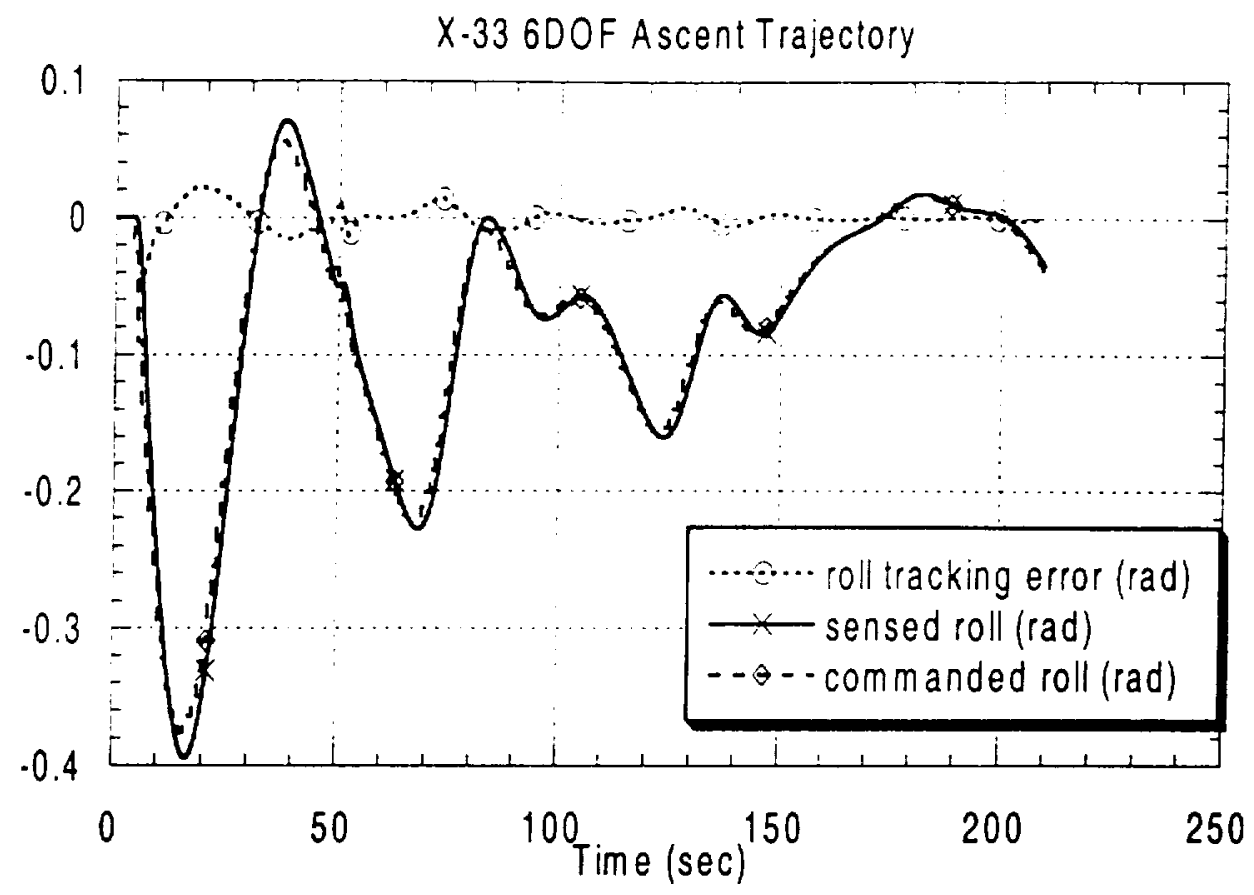

Fig. 3 Roll profile tracking

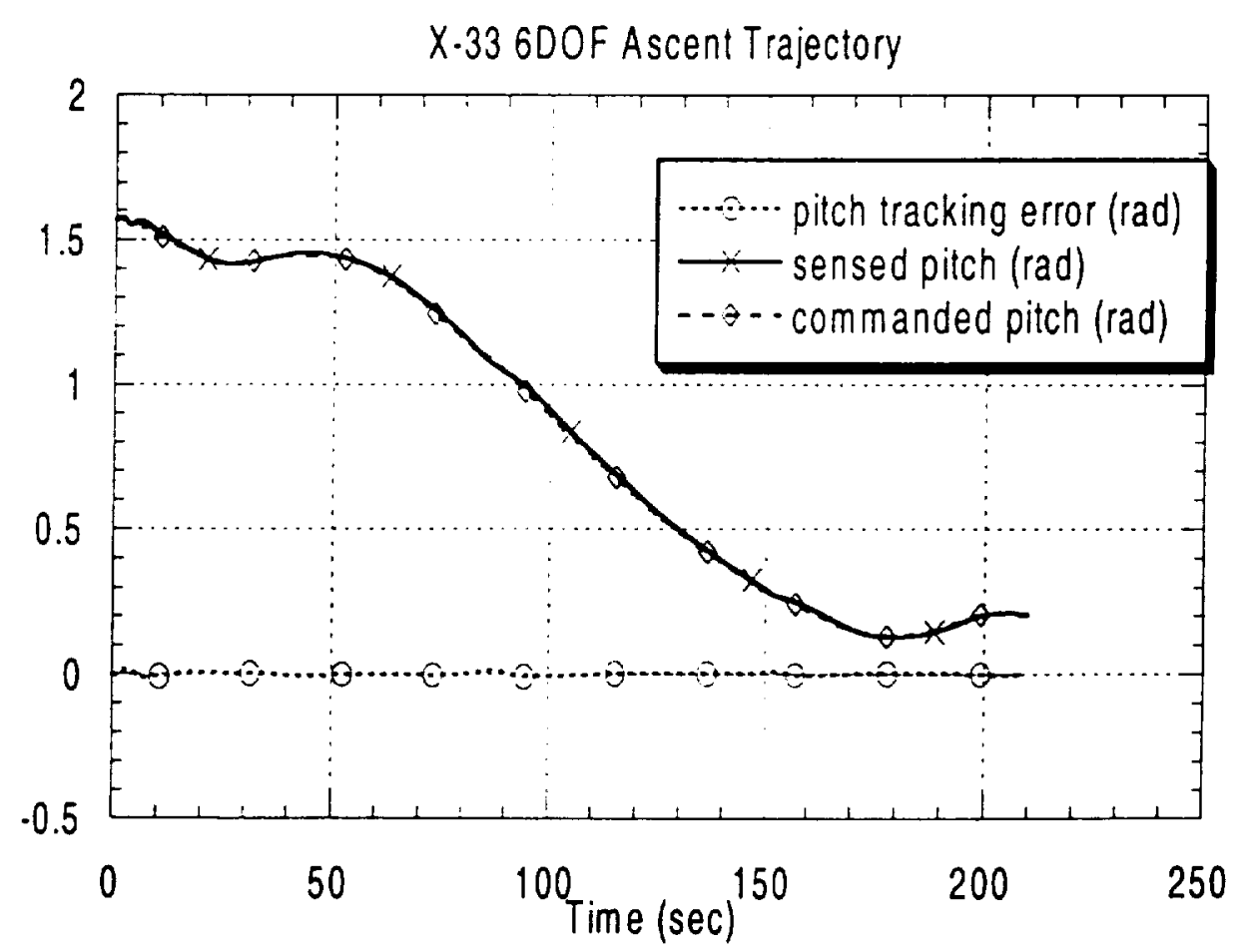

Fig. 4 Pitch profile tracking 


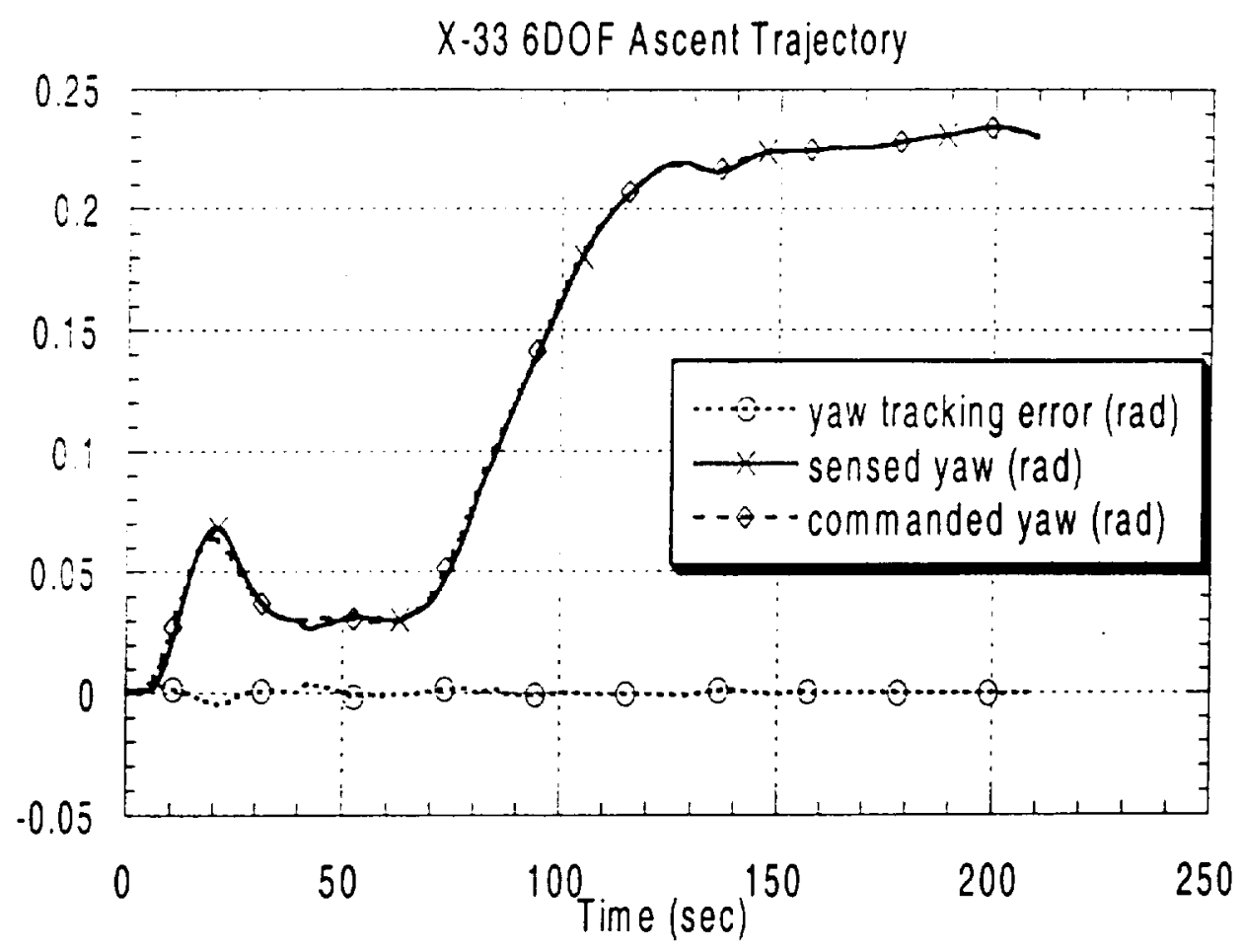

Fig. 5 Yaw profile tracking

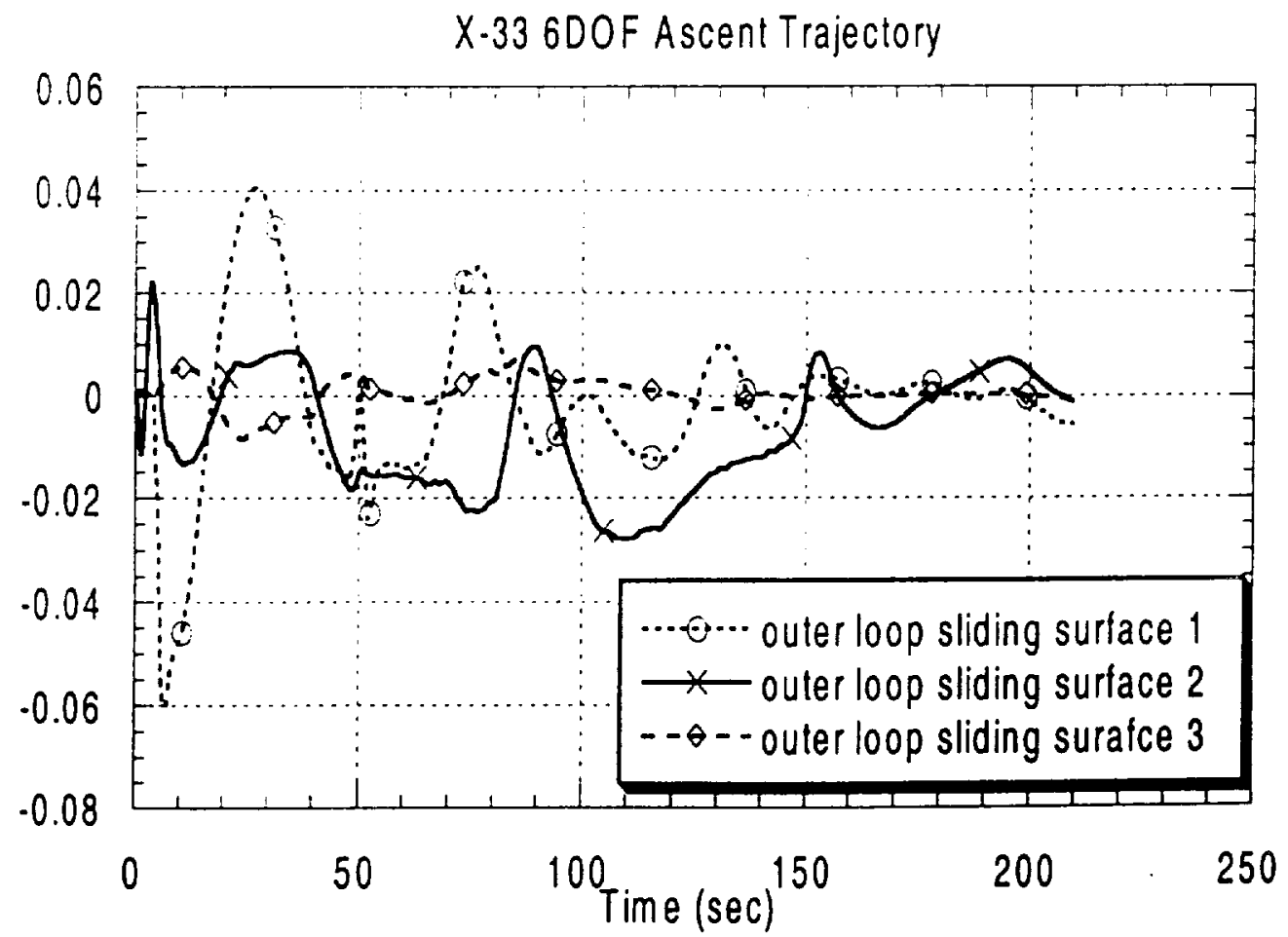

Fig. 6 Outer loop sliding surfaces 


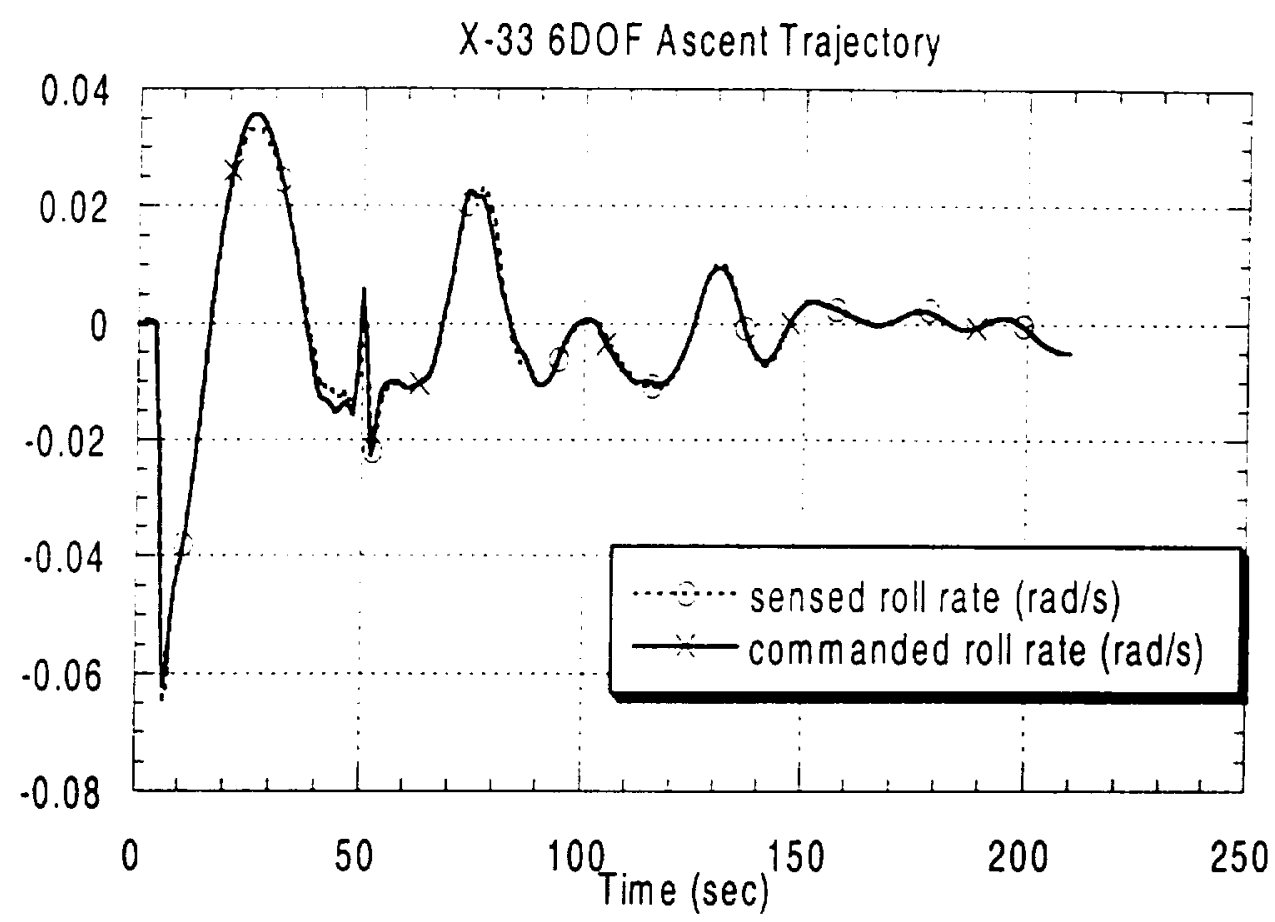

Fig. 7 Roll rate command tracking

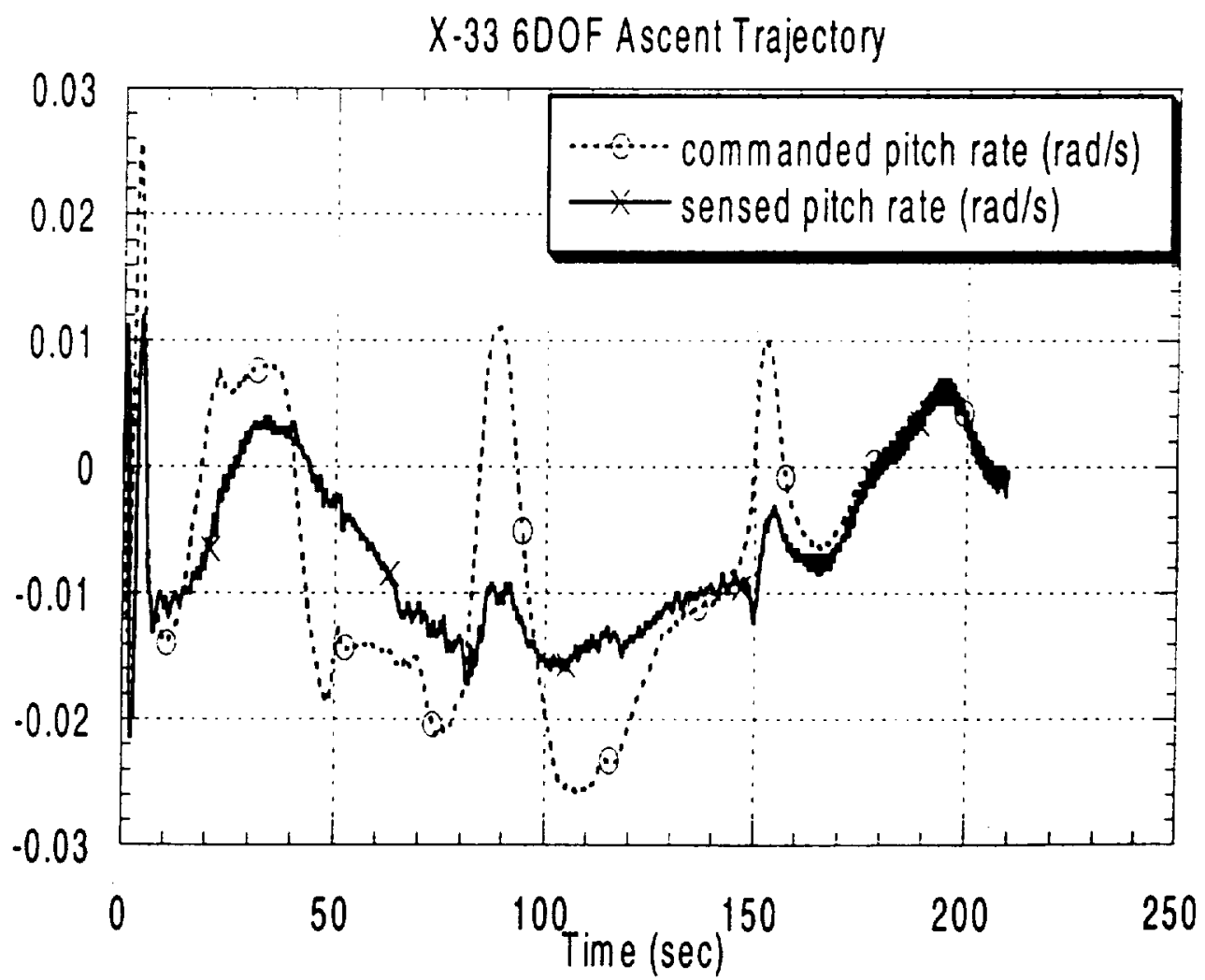

Fig. 8 Pitch rate command tracking 


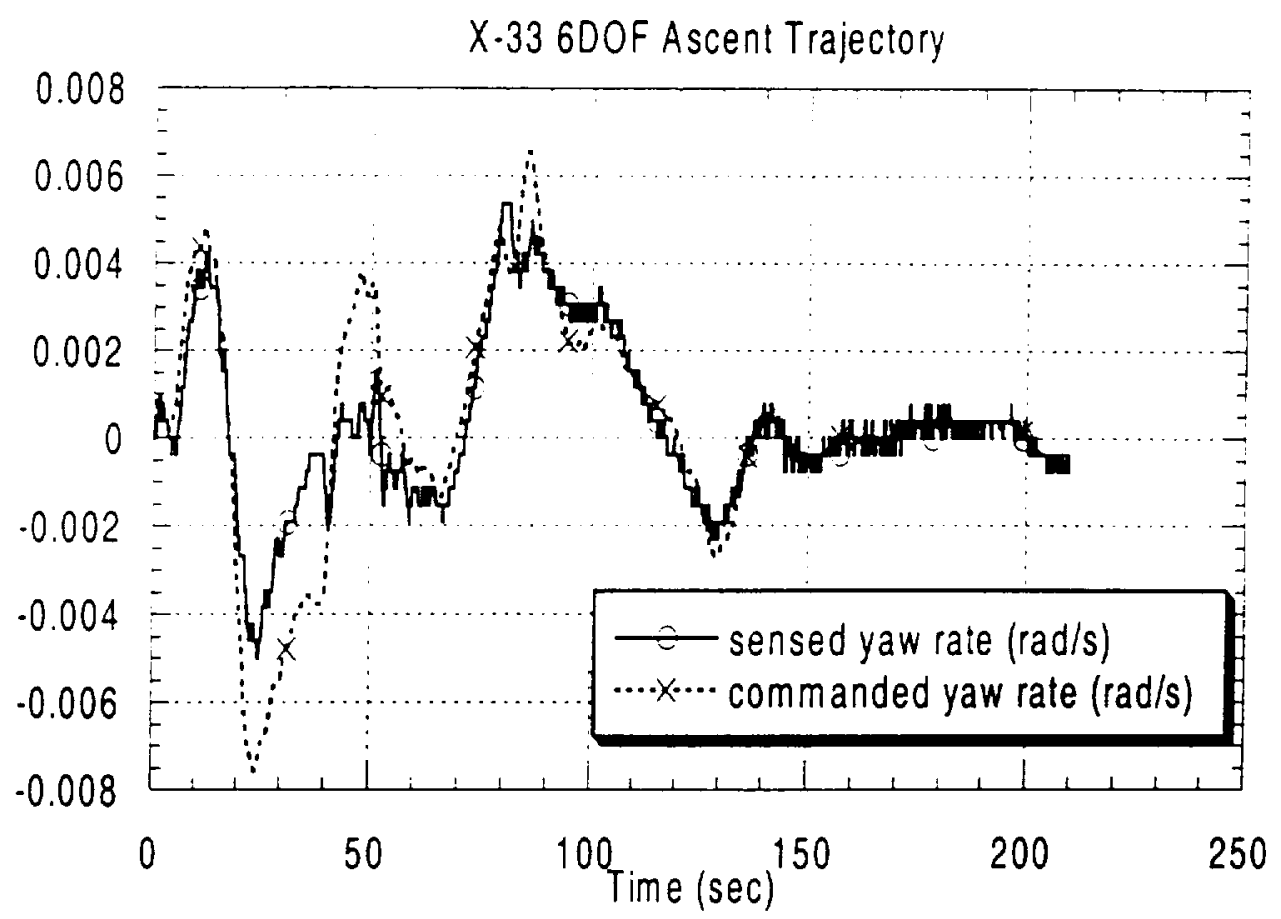

Fig. 9 Yaw rate command racking

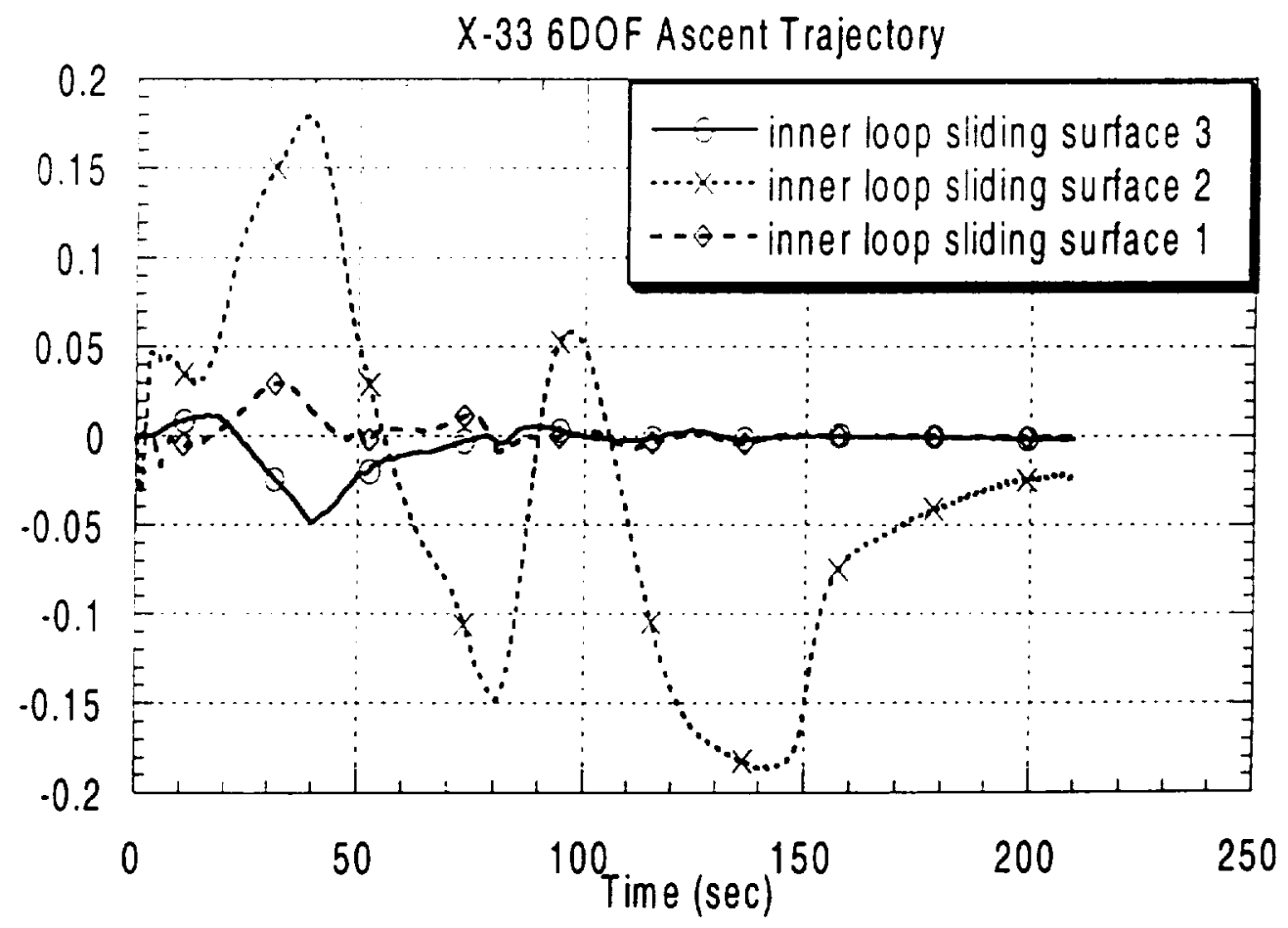

Fig. 10 Inner loop sliding surfaces 


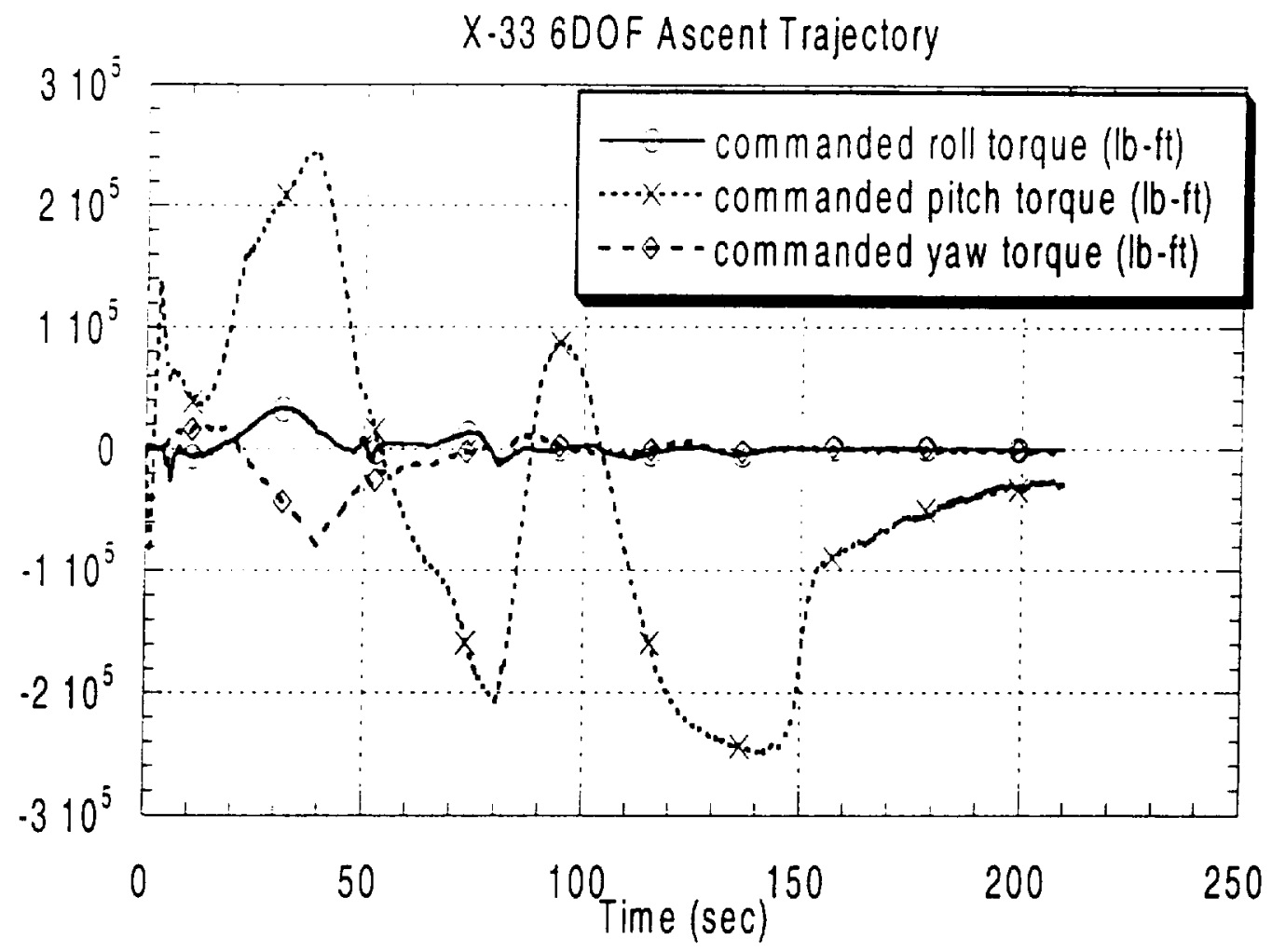

Fig. 11 Roll, Pitch and Yaw commanded torque (lb-ft)

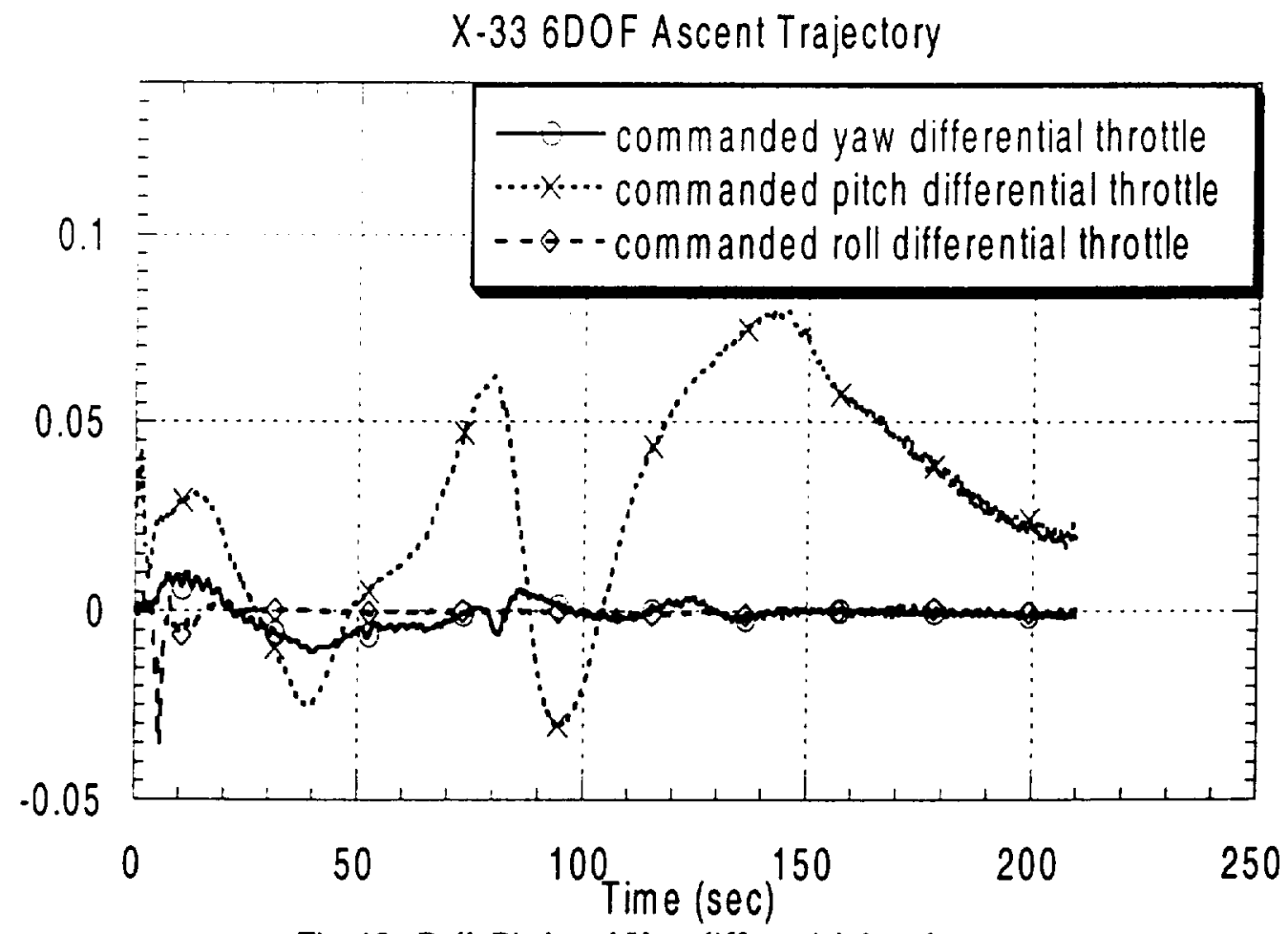

Fig. 12 Roll, Pitch and Yaw differential throttle 


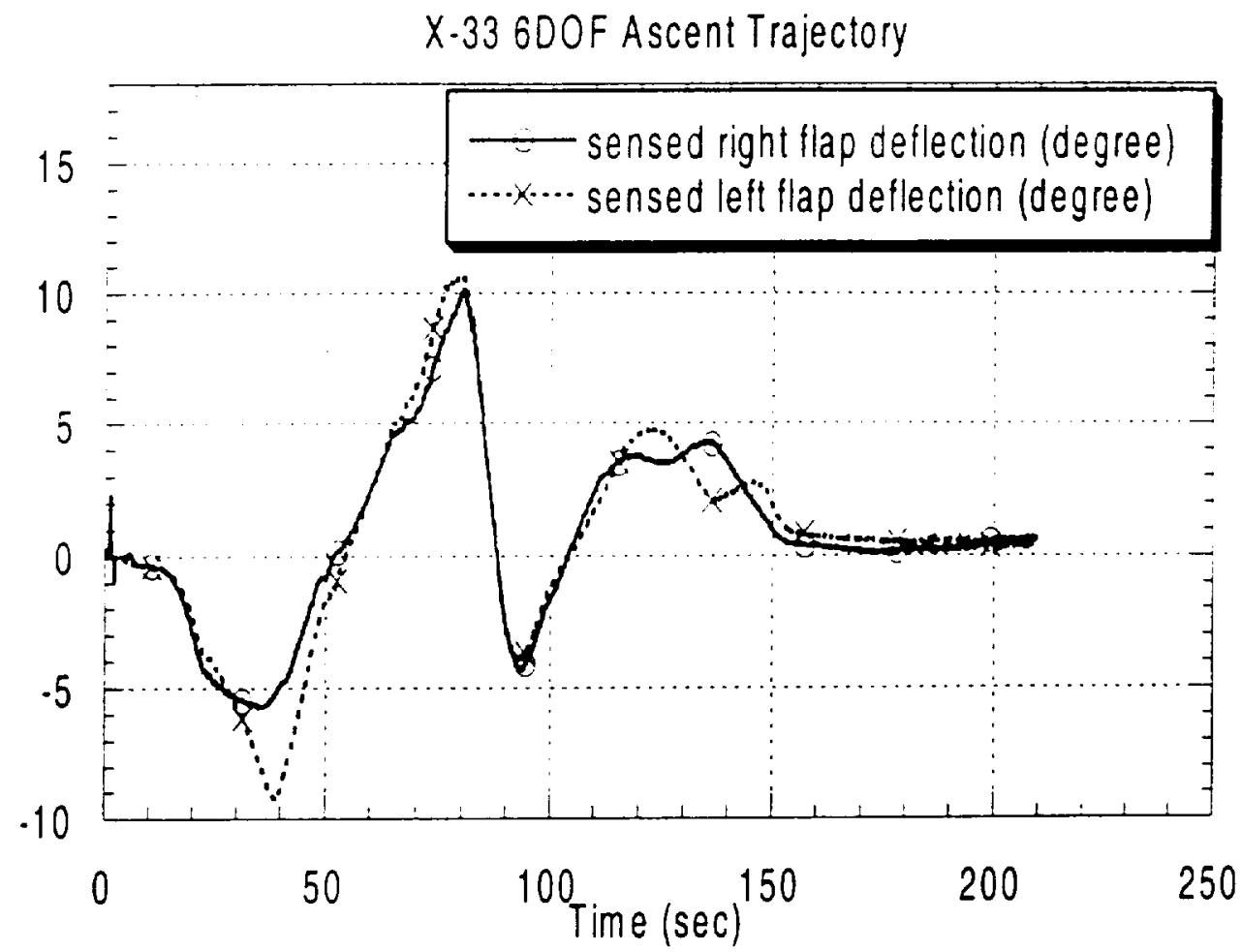

Fig. 13 Sensed tlap deflections

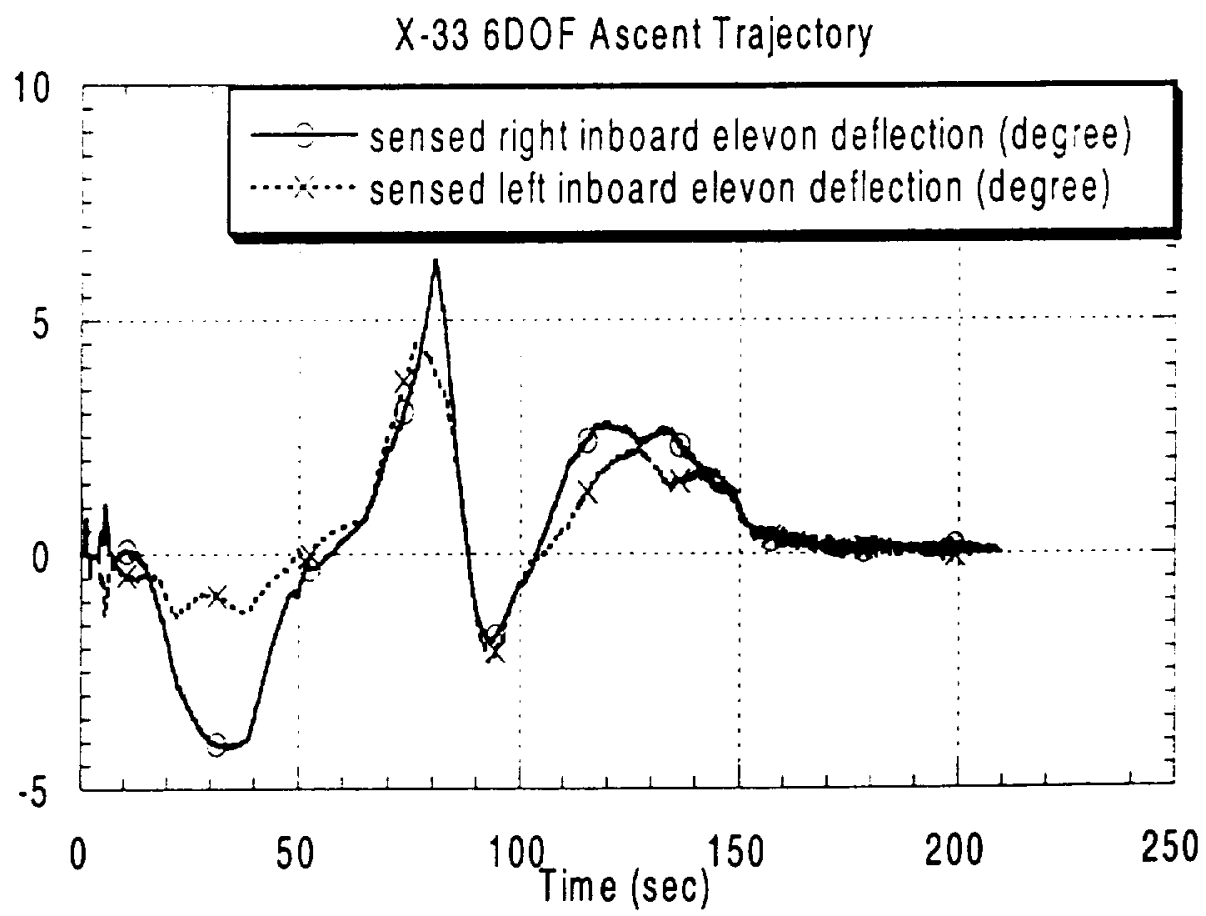

Fig. 14 Sensed inboard elevon deflections 


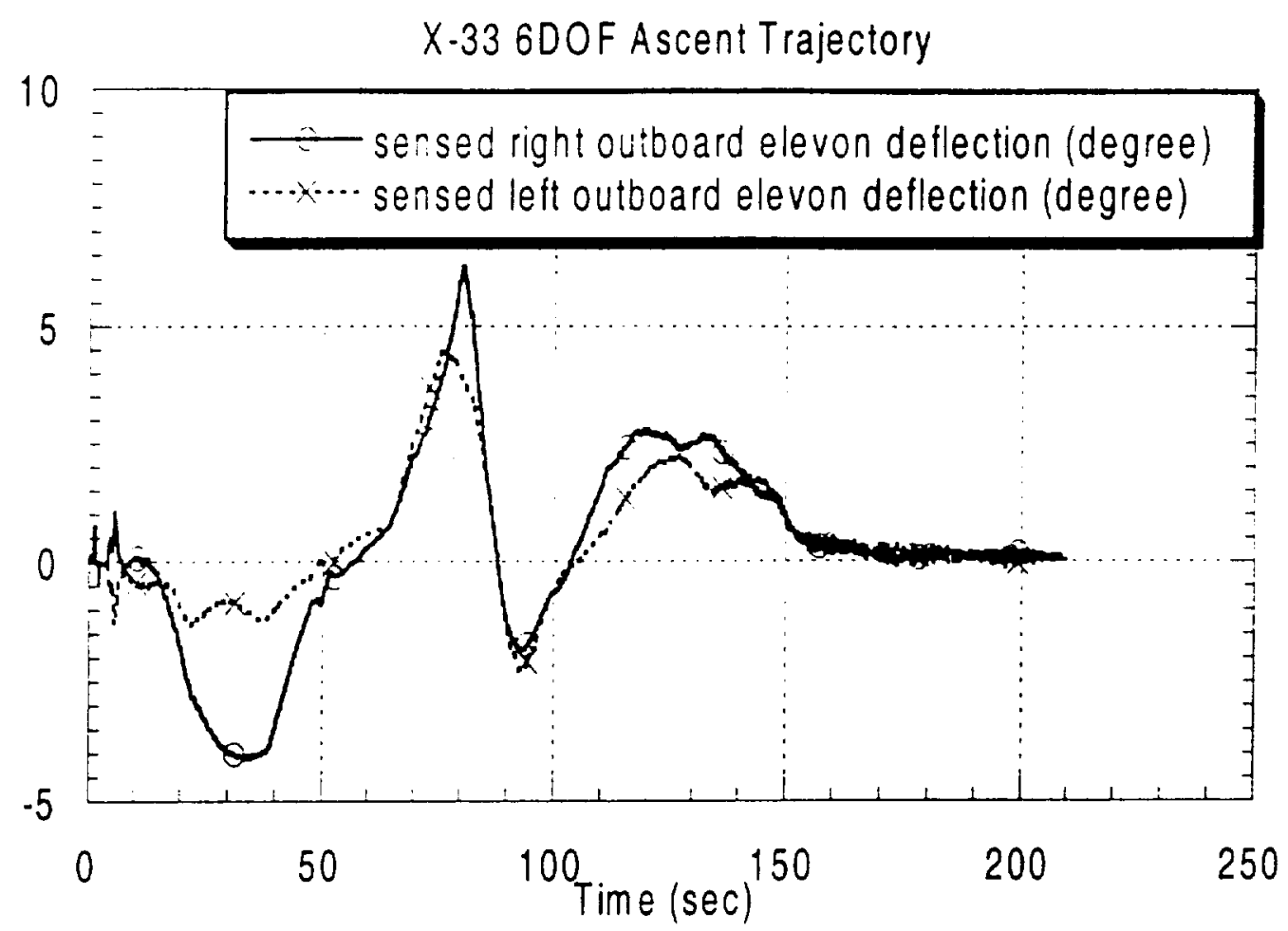

Fig. 15 Sensed outboard elevon deflections

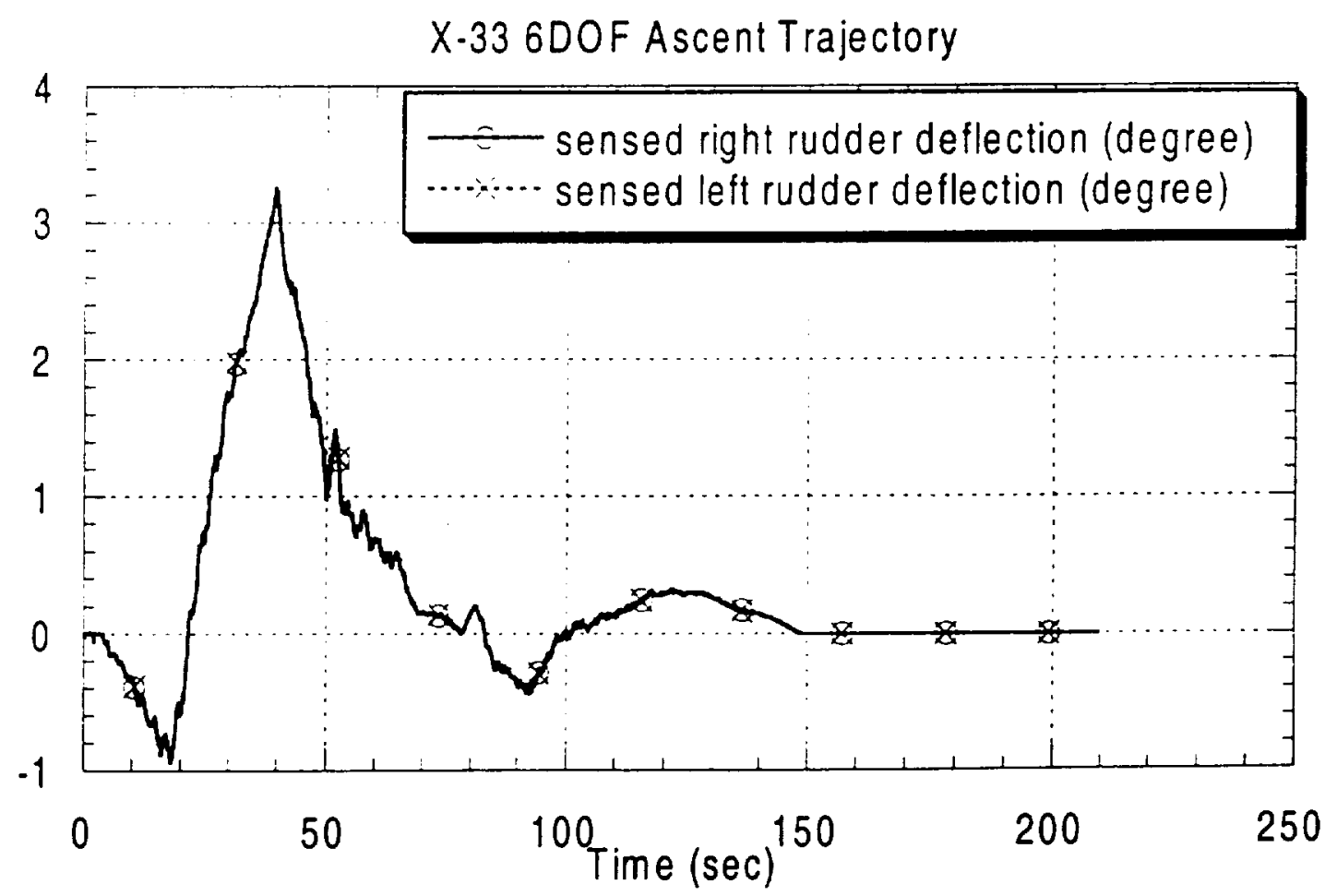

Fig. 16 Sensed rudder deflections 


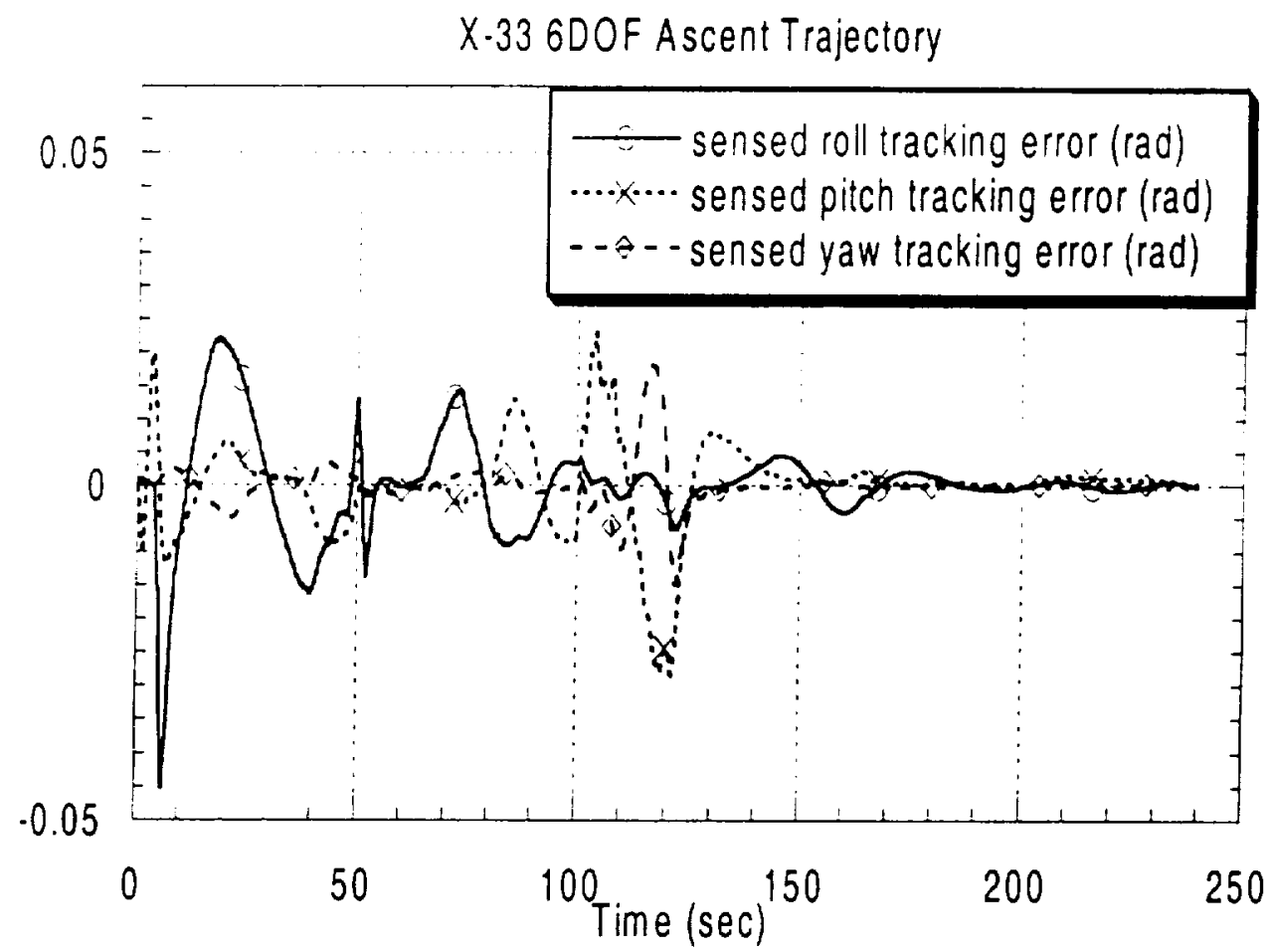

Fig. 17 Sensed orientation angle tracking errors given power pack out at $100 \mathrm{sec}$. 